\title{
Influence of gender and women's age on the prevalence of parathyroid failure after total thyroidectomy for multinodular goiter
}

\author{
Inés Villarroya-Marquina ${ }^{1,2}$, Leyre Lorente-Poch ${ }^{1,2}$, Juan Sancho $^{1,2}$, Antonio Sitges-Serra $^{1,2}$ \\ ${ }^{1}$ Endocrine Surgery Unit, Hospital del Mar, Barcelona, Spain; ${ }^{2}$ Departament de Cirurgia, Universitat Autònoma de Barcelona, Barcelona, Spain \\ Contributions: (I) Conception and design: All authors; (II) Administrative support: I Villarroya-Marquina, L Lorente-Poch; (III) Provision of study \\ materials or patients: L Lorente-Poch, J Sancho, A Sitges-Serra; (IV) Collection and assembly of data: I Villarroya-Marquina, L Lorente-Poch; (V) \\ Data analysis and interpretation: All authors; (VI) Manuscript writing: All authors; (VII) Final approval of manuscript: All authors. \\ Correspondence to: Prof. Antonio Sitges-Serra, MD, FRCS. Endocrine Surgery Unit, Hospital del Mar, Passeig Marítim 25-29, 08003 Barcelona, \\ Spain. Email: asitges@hospitaldelmar.cat.
}

\begin{abstract}
Backgroundk Female gender, particularly of a young age, has been reported as a risk factor for hypocalcemia after total thyroidectomy. There are no studies, however, addressing specifically the influence of women's age and menstrual status on postoperative parathyroid function.

Methods: Cohort study of consecutive patients undergoing total thyroidectomy for benign goiter between 2000-2017, excluding those with associated hyperparathyroidism, reoperation or conservative procedures. Prevalence of postoperative hypocalcemia (s-Ca $<8 \mathrm{mg} / \mathrm{dL}$ at 24 hours), protracted (1-month) and permanent hypoparathyroidism ( $>1$ year) were the main variables studied. Complete $>1$-year follow-up was achieved for all patients developing post-thyroidectomy hypocalcemia. Demographic, disease-related, number of parathyroid glands remaining in situ (PGRIS), biochemical and surgical variables were recorded. The impact of menstrual status on parathyroid function was analyzed by comparing two groups of women using a cut-off age of 45 years.
\end{abstract}

Results: A total of 811 patients were included: 14 percent were males and 86 percent females with a mean age of 53.2 years. The prevalence of postoperative hypocalcemia was ten points higher in women than in men $(23.7 \%$ vs. $36.4 \%$; $\mathrm{P}=0.008)$. Permanent hypoparathyroidism was more common in women than in men ( $5 \%$ vs. $0.9 \% ; \mathrm{P}=0.048$ ). Compared to females $\geq 45$ years, young women presented higher rates of all three parathyroid failure syndromes despite similar PGRIS scores. Age $<45$ years and low PGRIS scores were the only independent variables predicting postoperative hypocalcemia in females.

Conclusions: Premenopausal patients presented a higher prevalence of parathyroid failure and permanent hypoparathyroidism with similar PGRIS scores suggesting the presence of a sex-hormone factor influencing post-thyroidectomy parathyroid function.

Keywords: Hypocalcemia; hypoparathyroidism; menopause; parathyroid glands remaining in situ (PGRIS); prevalence; women

Submitted Oct 30, 2019. Accepted for publication Jan 04, 2020.

doi: 10.21037 /gs.2020.02.01

View this article at: http://dx.doi.org/10.21037/gs.2020.02.01

\section{Introduction}

Total thyroidectomy implies a significant traumatic aggression to the parathyroid glands. With the increased use of this surgical technique for a variety of benign and malignant disorders, postoperative hypocalcemia has become the most common complication following total thyroidectomy with a prevalence ranging from $19 \%$ to $38 \%$ (1). Acute parathyroid insufficiency due to gland devascularization, 
autotransplantation or inadvertent gland resection is currently considered the main, if not the only, cause of postthyroidectomy hypocalcemia. Our group previously reported that the number of parathyroid glands remaining in situ (PGRIS) - not autotransplanted nor inadvertently excised- is a key factor for the development of parathyroid insufficiency after total thyroidectomy (2). Additionally, biochemical and clinical factors such as preoperative calcium levels, perioperative PTH levels, hyperthyroidism, reoperation and female gender have been occasionally associated with postoperative hypocalcemia (3-5).

Several studies have reported that female gender is a risk factor for post-thyroidectomy hypocalcemia $(1,2,5,6)$. Edafe et al. (1) carried out a metanalysis with a total of 3,443 patients, examining gender as a predictor of hypocalcemia, and found that women had a significantly higher prevalence of postoperative parathyroid failure (OR 2.8). The authors hypothesized that this could be related to female patients having lower circulating levels of vitamin $\mathrm{D}$. The role of vitamin D status on postoperative parathyroid function, however, is far from clear with a single study suggesting an influence of low circulating vitamin D (7) and several others not reproducing these findings (8-10). In addition, this metanalysis emphasized the role of both accidental parathyroidectomy and autotransplantation as predictors of postoperative hypocalcemia. It did not assess, however, a possible difference in hypocalcemia rates between preand postmenopausal women. In fact, studies evaluating the influence of age or menstrual status on postoperative hypocalcemia in female patients are not available.

In the present work, we aimed to further analyze the impact of age, PGRIS score and pathology on parathyroid insufficiency after total thyroidectomy in female patients. In order to analyze a homogenous series of patients and to avoid confounding factors (bone hunger, lymphadenectomies, extended resections), we decided to analyze only total thyroidectomies for multinodular goiter.

\section{Methods}

\section{Study design}

Review of a prospectively maintained clinical database of a cohort of consecutive adult ( $>18$ years) patients who underwent first-time total thyroidectomy for multinodular goiter between 2000 and 2017 at the Endocrine Surgery Unit of the Hospital del Mar, a tertiary teaching hospital in Barcelona, Spain. The same experienced team of surgeons performed all the thyroidectomies. Exclusion criteria were reoperations, associated parathyroidectomy for primary or secondary hyperparathyroidism, Dunhill procedures for asymmetrical goiter, thyroid carcinoma and Graves' disease (usually treated with less than total surgical techniques). The prevalence of postoperative, protracted and permanent hypoparathyroidism was investigated. Demographic, disease-related, PGRIS score (see below), biochemical and surgical variables were recorded. A cut-off at 45 years was chosen to assess differences between pre- and post-menopausal women since the precise age at menopause was not recorded before 2017 .

\section{Surgical technique}

Total extracapsular thyroidectomy was performed in all cases. Parathyroid glands were sought in their usual position and no effort was made to identify them when located in non-orthotopic places. The identification of parathyroid glands was based solely on visual macroscopic features. Ligation of the inferior thyroid artery was carried out at the level of distal branches and close to the thyroid gland to minimize the devascularization of the parathyroid glands. Parathyroid autotransplantation was performed when glands were completely devascularized or accidentally excised and spotted before sending the specimen to the pathology lab.

\section{Definitions}

Parathyroid failure syndromes were defined in line with prior reports (11). Postoperative hypocalcemia was defined as a serum calcium levels $<8 \mathrm{mg} / \mathrm{dL}(2 \mathrm{mmol} / \mathrm{L}) 24$ hours and/or a PTH concentration $<10 \mathrm{pg} / \mathrm{mL} 4$ hours after thyroidectomy; protracted hypoparathyroidism as iPTH levels lower than $13 \mathrm{pg} / \mathrm{mL}$ and/or the need for calcium \pm vitamin $\mathrm{D}$ supplements 1 month after surgery; and permanent hypoparathyroidism was considered when PTH levels were lower than $13 \mathrm{pg} / \mathrm{mL}$ and/or replacement therapy was still required 1 year after surgery (12). The number of PGRIS was calculated as PGRIS $=4-$ (glands autotransplanted + inadvertently resected) (2). Patients were classified according to the PGRIS score as 1-2 (one or two PGRIS), 3 (three PGRIS) or 4 (all four glands remaining in situ). Patients' consent to use anonymized data for this observational clinical research was obtained together with the informed consent for thyroidectomy.

\section{Patient management}

Selective replacement therapy with calcium and calcitriol 


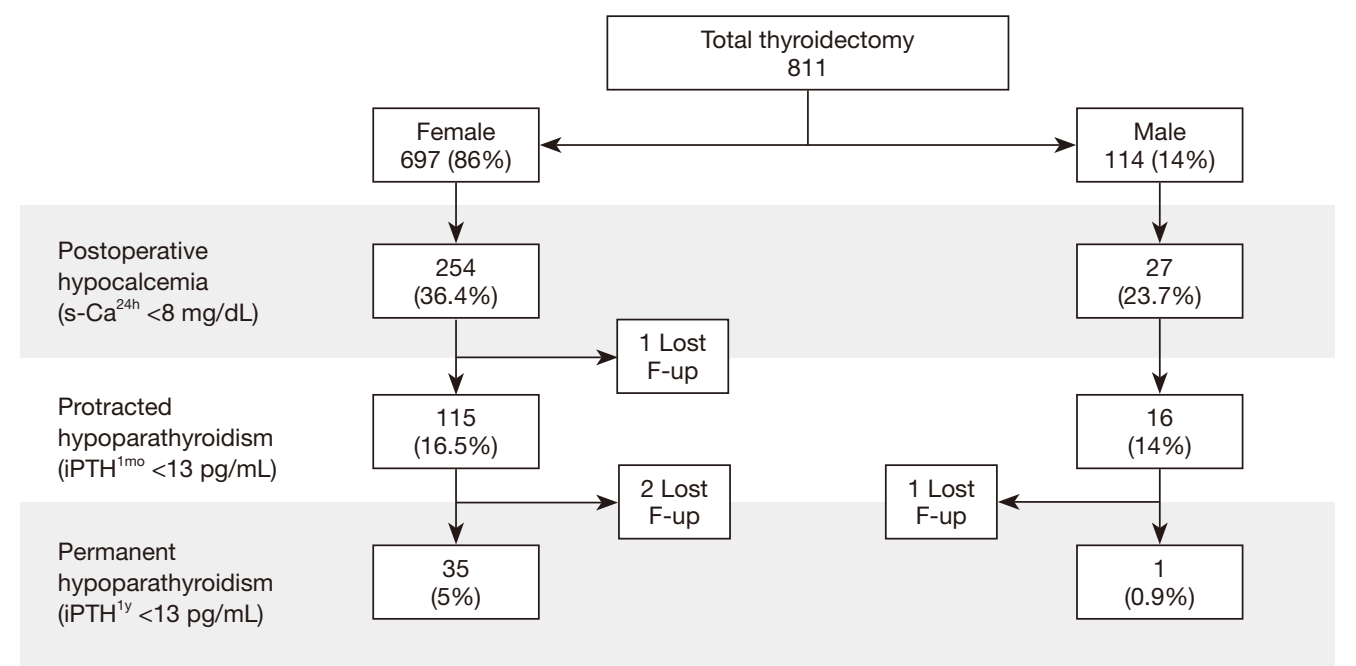

Figure 1 Flowchart describing the calcemic and parathyroid post-surgical evolution of the 811 total thyroidectomy patients included in the analysis classified by gender. Patients lost in follow-up are shown as well.

$(1.5-3 \mathrm{~g} /$ day of calcium carbonate and $0.5-1.5 \mu \mathrm{g} /$ day of calcitriol) was started in patients with low iPTH $(<10 \mathrm{pg} / \mathrm{mL})$ at 4 hours after surgery or s- $\mathrm{Ca}<8 \mathrm{mg} / \mathrm{dL}$ at 24 hours after thyroidectomy.

Patients were discharged on the first or second postoperative day and they were followed in the outpatient clinic. In patients with postoperative hypocalcemia, iPTH and serum calcium were measured at regular intervals until recovery or diagnosis of permanent hypoparathyroidism, after at least 1-year follow-up (12). Weaning off replacement therapy was initiated when serum calcium concentration was $\geq 8 \mathrm{mg} / \mathrm{dL}$ and iPTH showed a sustained increase up to normal serum concentrations.

Serum iPTH levels were determined using either an immunoradiometric second-generation assay using an iPTH IRMA assay (Scantibodies Laboratory, Santee, CA, USA) or a solid-phase, two-site chemiluminescent enzyme-labeled assay, IMMULITE 2000 Intact PTH assay (Siemens Healthcare Diagnostics Spain, Madrid, Spain). The normal range was $13-65 \mathrm{pg} / \mathrm{mL}$ and the detection limit $3 \mathrm{pg} / \mathrm{mL}$ (1.4-7.5 pmol/L, detection limit $0.4 \mathrm{pmol} / \mathrm{L})$. Thyroidectomy specimens were processed by the same dedicated pathologist who consistently reported on the parathyroid glands inadvertently excised.

\section{Statistical analysis}

The main outcome variable of the study was the prevalence of postoperative hypocalcemia, protracted and permanent hypoparathyroidism in relation to gender and female age. Data was collected using a database created with FileMaker ${ }^{\circledR}$ Pro-advance 13.0 v4 software (File-Maker, Santa Clara, CA, USA) and extracted from the clinical records from the hospital's general database. Demographic, clinical, surgical, pathological and biochemical variables relevant to the diagnosis and management of postoperative hypocalcemia were included.

Statistical analyses were done using SPSS $^{\circledR}$ version 23.0 (IBM, Armonk, NY, USA). The normal distribution of quantitative variables was assessed with the KolmogorovSmirnov test. Quantitative continuous and normally distributed variables are expressed as mean (SD) and compared using Student's $t$-test for unpaired samples or the ANOVA test. Non-parametric tests (Mann-Whitney $\mathrm{U}$ test and Kruskal-Wallis test) were used when required. Dichotomous variables were compared using the Chisquare or Fisher's exact test as appropriate. Binomial logistic regression analysis with predictors selected by a forward stepwise procedure was used to assess the risk factors for postoperative parathyroid failure among women. Statistical significance (two-tailed) was set at $\mathrm{P}<0.05$.

\section{Results}

Some 811 total thyroidectomy patients were included in the analysis (Figure 1). There were 114 men (14\%) and 697 women $(86 \%)$ with a mean age of 53.2 years. The prevalence of postoperative hypocalcemia was $23.7 \%$ 
Table 1 Parathyroid failure syndromes, biochemical and clinical variables after total thyroidectomy in male and female patients.

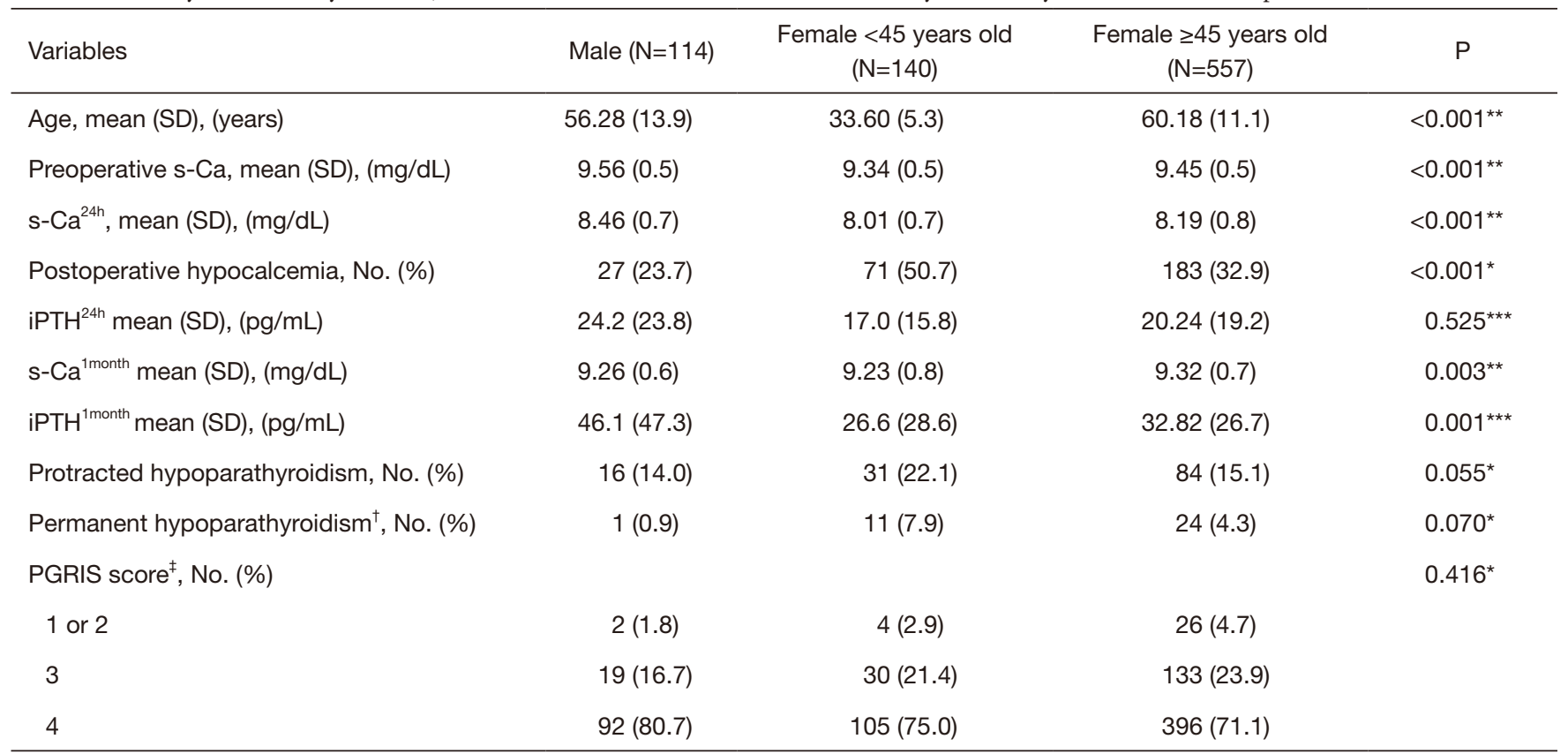

${ }^{\star}$, Chi-squared test; ${ }^{\star \star}$, one-way ANOVA test; ${ }^{\star \star \star}$, Kruskal-Wallis test; ${ }^{\dagger}$, data not available for one male patient and one female patient; ${ }^{\ddagger}$, data not available for two female patients. PGRIS: parathyroid glands remaining in situ.

in males and $36.4 \%$ in women $(\mathrm{P}=0.008)$. Permanent hypoparathyroidism was more common in women $(5 \% v s$. $0.9 \% ; \mathrm{P}=0.047)$.

The gender differences in clinical and biochemical parameters are shown in Table 1. The mean of PGRIS scores were similar in males and females $[3.7$ (0.6) vs. 3.6 (0.6); $\mathrm{P}=0.484]$. Thus, different rates of postoperative hypocalcemia between men and women cannot be attributed to variations in the intraoperative management of the parathyroid glands.

Regarding just female patients, hypocalcemia developed in half of those aged $<45$ years whereas it was documented in only one-third of those aged $\geq 45$ years. Accordingly, young women showed a higher prevalence of protracted and permanent hypoparathyroidism. Although PTH concentration at 24 hours after surgery was not different, serum calcium levels were significantly lower in young patients.

To adjust for the intraoperative management of the parathyroid glands, a final analysis was performed in cases where the four glands were left in situ (PGRIS 4). Women $<45$ years exhibited a worse biochemical and clinical profile than those aged 45 years or more with over a two-fold prevalence of permanent hypoparathyroidism (Table 2).
A binomial logistic regression multivariate analysis of variables protecting from postoperative hypocalcemia in females, showed that the only independent factors were preoperative serum calcium OR: 0.5 (95\% CI: 0.35-0.82), age $>45$ years with OR: 0.6 (95\% CI: $0.39-0.96)$ and high PGRIS (PGRIS 3-4) scores with OR: 0.6 (95\% CI: $0.45-$ $0.76)$.

\section{Discussion}

Patients' age and gender are among the few clinical variables traditionally associated with hypocalcemia after total thyroidectomy $(2,7-10,13)$. Some authors have even suggested that replacement therapy should be given to all female patients undergoing total thyroidectomy even if three parathyroid glands were correctly preserved (14). On the other hand, several reports have not identified an increased risk for hypocalcemia in women. Most of these, however, are flawed by the inclusion of conservative surgical procedures, different definitions of postoperative hypocalcemia and lack of long-term follow-up (15-17).

The present study focused on a homogeneous series of thyroidectomies for multinodular goiter, confirms the higher prevalence of postoperative parathyroid failure in 
Table 2 Parathyroid failure syndromes after total thyroidectomy in women with four parathyroid glands remaining in situ (PGRIS 4)

\begin{tabular}{|c|c|c|c|}
\hline Variables & $<45$ years old $(n=105)$ & $\geq 45$ years old $(n=396)$ & $\mathrm{P}$ \\
\hline Preoperative s-Ca, mean (SD), (mg/dL) & $9.29(0.4)$ & $9.49(0.4)$ & $<0.001^{* *}$ \\
\hline $\mathrm{s}-\mathrm{Ca}^{24 \mathrm{~h}}$, mean $(\mathrm{SD}),(\mathrm{mg} / \mathrm{dL})$ & $8.03(0.7)$ & $8.34(0.7)$ & $<0.001^{\star \star}$ \\
\hline Postoperative hypocalcemia, No. (\%) & $49(46.7)$ & $102(25.8)$ & $<0.001^{*}$ \\
\hline $\mathrm{s}-\mathrm{Ca}{ }^{1 \mathrm{month}}$, mean $(\mathrm{SD}),(\mathrm{mg} / \mathrm{dL})$ & $9.11(0.7)$ & $9.36(0.6)$ & $0.004^{\star \star}$ \\
\hline $\mathrm{iPTH}^{1 \text { month }}$, mean (SD), (pg/mL) & $30.44(26.5)$ & $37.58(27.4)$ & $0.051^{\star * *}$ \\
\hline Protracted hypoparathyroidism, No. (\%) & $20(19.0)$ & $38(9.6)$ & $0.007^{*}$ \\
\hline Permanent hypoparathyroidism, No. (\%) & $7(6.7)$ & $9(2.3)$ & $0.022^{*}$ \\
\hline
\end{tabular}

${ }^{*}$, Chi-squared test; ${ }^{* *}$, Students $t$-test; ${ }^{* \star}, \mathrm{U}$ de Mann-Whitney. iPTH, intact parathyroid hormone.

women and further deepens into the issue by adding to the analysis the presumed menopausal status and the number of PGRIS, a key variable influencing the postoperative parathyroid function $(2,18)$.

Sands et al. (6) were the first to address in some detail the issue of female gender as a risk factor for postthyroidectomy hypocalcemia and reported that women showed a 2.14 relative risk for this complication. However, they did not find a significant difference in rates of transient hypocalcemia between premenopausal and postmenopausal women using a cut-off age of 50 years. In this retrospective study of a small female cohort, the authors did not take into account the indication for surgery nor PGRIS. A high rate of inadvertent parathyroidectomy (one gland every two thyroidectomies in women) could have also influenced the results. We set the age cut-off to 45 years for two reasons: (I) this is the average age women become perimenopausal in Spain where mean age at full menopause in 51 years, and (II) a 5- to 6-year prior interval is a sound assumption based on FSH dynamics (19). Using this age cut-off, substantial and statistically significant differences were observed in all clinical and biochemical parameters of parathyroid function between both age groups. This assumption, however, limits the precision of our analysis and calls for further investigations in which the exact age at menopause should be recorded prospectively.

The present study reveals that half of the females under 45 years of age developed postoperative hypocalcemia compared to just one-third of those $>45$ years, regardless of similar concentration of iPTH at 24 hours after surgery and similar PGRIS scores. Furthermore, permanent hypoparathyroidism was more common in women than in men operated on for multinodular goiter. Gender differences were particularly noticed in young women in whom all parathyroid failure syndromes were more prevalent.

A final analysis adjusting for PGRIS 4, showed that age is a key variable for the development of postoperative parathyroid failure: despite proper preservation of the four parathyroid glands, women under 45 had a significantly worse biochemical and clinical profiles.

We can only speculate about the possible influence of circulating estrogens on the parathyroid function after thyroidectomy. Recently, Haglund et al. (20) have identified estrogens receptors (ERB1 and ERB2) in normal parathyroid cells. They also found that the ERB1 negative parathyroid adenomas had significantly higher adenoma weight, serum calcium, and iPTH serum concentration. This suggests a potential downregulating effect of estrogens on PTH synthesis and secretion, a pathophysiological event which would be supported also by animal studies pointing in this same direction (21). In addition, estrogens inhibit osteoclast activity and bone resorption and may render the bone more resistant to the effects of PTH.

\section{Conclusions}

The higher prevalence of post-thyroidectomy parathyroid failure in young females compared to both men and postmenopausal women appears to be independent of PGRIS and could be mediated by higher levels of circulating estrogens. Additional prospective studies, however, are 
needed to further elucidate the complex interaction between female sex hormones and postoperative parathyroid cell function.

\section{Acknowledgments}

Funding: None.

\section{Footnote}

Conflicts of Interest: All authors have completed the ICMJE uniform disclosure form (available at http://dx.doi. org/10.21037/gs.2020.02.01). ASS serves as the unpaid editorial board member of Gland Surgery from Mar 2018 to Feb 2023. The other authors have no conflicts of interest to declare.

Ethical Statement: The authors are accountable for all aspects of the work in ensuring that questions related to the accuracy or integrity of any part of the work are appropriately investigated and resolved. The study was approved by the institutional review board (2017/7557/I). Patients' consent to use anonymized data for this observational clinical research was obtained together with the informed consent for thyroidectomy.

Open Access Statement: This is an Open Access article distributed in accordance with the Creative Commons Attribution-NonCommercial-NoDerivs 4.0 International License (CC BY-NC-ND 4.0), which permits the noncommercial replication and distribution of the article with the strict proviso that no changes or edits are made and the original work is properly cited (including links to both the formal publication through the relevant DOI and the license). See: https://creativecommons.org/licenses/by-nc-nd/4.0/.

\section{References}

1. Edafe $\mathrm{O}$, Antakia R, Laskar N, et al, Balasubramanian SP. Systematic review and meta-analysis of predictors of postthyroidectomy hypocalcaemia. Br J Surg 2014;101:307-20.

2. Lorente-Poch L, Sancho JJ, Ruiz S, et al. Importance of in situ preservation of parathyroid glands during total thyroidectomy. Br J Surg 2015;102:359-67.

3. Barczyński M, Cichoń S, Konturek A. Which criterion of intraoperative iPTH assay is the most accurate in prediction of true serum calcium levels after thyroid surgery?. Langenbecks Arch Surg 2007;392:693-8.
4. Pesce CE, Shiue Z, Tsai HL, et al. Postoperative hypocalcemia after thyroidectomy for Graves' disease. Thyroid 2010;20:1279-83.

5. Thomusch O, Machens A, Sekulla C, et al. Multivariate analysis of risk factors for postoperative complications in benign goiter surgery: prospective multicenter study in Germany. World J Surg 2000;24:1335-41.

6. Sands NB, Payne RJ, Côté V, et al. Female gender as a risk factor for transient post-thyroidectomy hypocalcemia. Otolaryngol Head Neck Surg 2011;145:561-4.

7. Erbil Y, Bozbora A, Ozbey N, et al. Predictive value of age and serum parathormone and vitamin $\mathrm{d} 3$ levels for postoperative hypocalcemia after total thyroidectomy for nontoxic multinodular goiter. Arch Surg 2007;142:1182-7.

8. Press D, Politz D, Lopez J, et al. The effect of vitamin D levels on postoperative calcium requirements, symptomatic hypocalcemia, and parathormone levels following parathyroidectomy for primary hyperparathyroidism. Surgery 2011;150:1061-8.

9. Raffaelli M, De Crea C, D'Amato G, et al. Postthyroidectomy hypocalcemia is related to parathyroid dysfunction even in patients with normal parathyroid hormone concentrations early after surgery. Surgery 2016;159:78-84.

10. Manzini G, Malhofer F, Weber T. Can preoperative vitamin $\mathrm{D}$ deficiency predict postoperative hypoparathyroidism following thyroid surgery?. Langenbecks Arch Surg 2019;404:55-61.

11. Lorente-Poch L, Sancho JJ, Muñoz-Nova JL, et al. Defining the syndromes of parathyroid failure after total thyroidectomy. Gland Surg 2015;4:82-90.

12. Villarroya-Marquina I, Sancho J, Lorente-Poch L, et al. Time to parathyroid function recovery in patients with protracted hypoparathyroidism after total thyroidectomy. Eur J Endocrinol 2018;178:103-11.

13. Lorente-Poch L, Sancho J, Muñoz JL, et al. Failure of fragmented parathyroid gland autotransplantation to prevent permanent hypoparathyroidism after total thyroidectomy. Langenbecks Arch Surg 2017;402:281-7.

14. Bove A, Bongarzoni G, Dragani G, et al. Should female patients undergoing parathyroid-sparing total thyroidectomy receive routine prophylaxis for transient hypocalcemia?. Am Surg 2004;70:533-6.

15. Erbil Y, Ozbey NC, Sari S, et al. Determinants of postoperative hypocalcemia in vitamin D-deficient Graves' patients after total thyroidectomy. Am J Surg 2011;201:685-91.

16. Thomusch O, Machens A, Sekulla C, et al. The impact of 
surgical technique on postoperative hypoparathyroidism in bilateral thyroid surgery: a multivariate analysis of 5846 consecutive patients. Surgery 2003;133:180-5.

17. Wilson RB, Erskine C, Crowe PJ. Hypomagnesemia and hypocalcemia after thyroidectomy: prospective study. World J Surg 2000;24:722-6.

18. Ponce de León-Ballesteros G, Velázquez-Fernández D, Hernández-Calderón FJ, et al. Hypoparathyroidism After Total Thyroidectomy: Importance of the Intraoperative Management of the Parathyroid Glands. World J Surg 2019;43:1728-35.

Cite this article as: Villarroya-Marquina I, Lorente-Poch L, Sancho J, Sitges-Serra A. Influence of gender and women's age on the prevalence of parathyroid failure after total thyroidectomy for multinodular goiter. Gland Surg 2020;9(2):245251. doi: $10.21037 /$ gs.2020.02.01
19. Sowers MR, Zheng H, McConnell D, et al. Follicle stimulating hormone and its rate of change in defining menopause transition stages. J Clin Endocrinol Metab 2008;93:3958-64.

20. Haglund F, Rosin G, Nilsson IL, et al. Tumour nuclear oestrogen receptor beta 1 correlates inversely with parathyroid tumour weight. Endocr Connect 2015;4:76-85.

21. Carrillo-López N, Román-García P, Rodríguez-Rebollar $\mathrm{A}$, et al. Indirect regulation of PTH by estrogens may require FGF23. J Am Soc Nephrol 2009;20:2009-17. 